\title{
The method of firefighters real-time locating based on RFID
}

\author{
Xiaohui Zeng ${ }^{1, a}$, Jianhua Jiang ${ }^{2, b}$ BaoYong Cheng ${ }^{3, c}$ \\ ${ }^{1}$ National institute of education, NanChang institute of Science \& Technology, \\ NanChang, 330108, China \\ ${ }^{2}$ National institute of education, NanChang institute of Science \& Technology, \\ NanChang, 330108, China \\ ${ }^{3}$ National institute of education, NanChang institute of Science \& Technology, \\ NanChang, 330108, China \\ aemail: 007_master@163.com, bemail:598225090@qq.com, c58404029@qq.com
}

Keywords: positioning;Range;RSSI;Coordinate estimate;fire;TOF;RFID

\begin{abstract}
This paper proposes a fireman positioning method based on RFID technology, which combined RSSI and TOF ranging in automatic positioning firefighters. When the system can't measure the distance due to various reasons, it will estimate the target location of the current based on historical data. The experimental results show that the method can position targets quickly and accurately. So it can be used in fighters positioning for the fire.
\end{abstract}

\section{Introduction}

In recent years, due to the increase of electric power, the number of fire disaster also presents the trend of increased year by year, and the fireman safety accidents also increased. In order to ensure the safety of firefighters, positioning firemen in the fire has become an important guarantee to protect the safety of firefighters ${ }^{[1]}$.

The WSN, GPS, the orientation of ZIGBEE, radar, WIFI and cellular networks are traditional non-visual positioning methods. They have a lot of shortcomings, such as high cost, lack of practical and the precision of positioning ${ }^{[2-6]}$. RFID technology has lots of advantages, such as the large range of transmission, high positioning accuracy, convenient installation, low cost and so on. In fire environments, it has obvious advantage.

In this paper, it proposes a fireman positioning method based on RFID technology, which combined RSSI and TOF ranging in automatic positioning firefighters ${ }^{[7]}$. When the system is able to obtain target location information, it determine the target location by using the least square method. In the process of operation, if the ranging failed occasionally, it will estimate the location of the object by using the trajectory fitting based on the previous data location.

\section{The spatial three-dimensional coordinates}

Assuming that there are several reference nodes in the three-dimensional space, $\mathrm{P}_{1}\left(\mathrm{a}_{1}, \mathrm{~b}_{1}, \mathrm{c}_{1}\right), \mathrm{P}_{2}$ $\left(a_{2}, b_{2}, c_{2}\right), \cdots \cdots P n(a n, b n, c n)$, moving in three-dimensional space coordinates of measuring points $P(x, y, z)$, and the distance between the reference node to the mobile node is $d_{1}, d_{2}, \ldots, d_{n}$ respectively. Then it can establish a specific $(\mathrm{x}, \mathrm{y}, \mathrm{z})$ and the reference node $\left(\mathrm{x}_{\mathrm{i}}, \mathrm{y}_{\mathrm{i}}, \mathrm{z}_{\mathrm{i}}\right)$ and their respective distance between equations based on the three dimensional space and distance formula. $(\mathrm{i}=1,2,3 \cdots \cdots \cdot \mathrm{n})$ :

$$
\left\{\begin{array}{c}
\left(x-a_{1}\right)^{2}+\left(y-b_{1}\right)^{2}+\left(z-c_{1}\right)^{2}=d_{1}^{2} \\
\left(x-a_{2}\right)^{2}+\left(y-b_{2}\right)^{2}+\left(z-c_{2}\right)^{2}=d_{2}^{2} \\
\vdots \\
\left(x-a_{n}\right)^{2}+\left(y-b_{n}\right)^{2}+\left(z-c_{n}\right)^{2}=d_{n}^{2}
\end{array}\right.
$$


Suppose $\mathrm{r}_{i}^{2}=d_{i}^{2}-\left(a_{i}^{2}+b_{i}^{2}+c_{i}^{2}\right)$, then subtract the behind equation from the before equation in type (1), and get the Nonhomogeneous linear equations by the simplify :

$$
\left\{\begin{array}{c}
\left(a_{2}-a_{1}\right) x+\left(b_{2}-b_{1}\right) y+\left(c_{2}-c_{1}\right) z=\frac{1}{2}\left(r_{1}^{2}-r_{2}^{2}\right) \\
\left(a_{3}-a_{2}\right) x+\left(b_{3}-b_{2}\right) y+\left(c_{3}-c_{2}\right) z=\frac{1}{2}\left(r_{2}^{2}-r_{3}^{2}\right) \\
\vdots \\
\left(a_{n}-a_{n-1}\right) x+\left(b_{n}-b_{n-1}\right) y+\left(c_{n}-c_{n-1}\right) z=\frac{1}{2}\left(r_{n-1}^{2}-r_{n}^{2}\right)
\end{array}\right.
$$

Make type (2) written in matrix form:

$$
\mathrm{Ax}=\mathrm{b}
$$

The coefficient matrix is: $A=\left[\begin{array}{ccc}a_{2}-a_{1} & b_{2}-b_{1} & c_{2}-c_{1} \\ a_{3}-a_{2} & b_{3}-b_{2} & c_{3}-c_{2} \\ \vdots & \vdots & \vdots \\ a_{n}-a_{n-1} & b_{n}-b_{n-1} & c_{n}-c_{n-1}\end{array}\right]$, And the unknown vectors is : $x=\left[\begin{array}{c}x \\ y \\ z\end{array}\right]$,

The product is: $\mathrm{b}=\frac{1}{2}\left[\begin{array}{c}\mathrm{r}_{1}^{2}-\mathrm{r}_{2}^{2} \\ \mathrm{r}_{2}^{2}-\mathrm{r}_{3}^{2} \\ \vdots \\ \mathrm{r}_{n-1}^{2}-\mathrm{r}_{n}^{2}\end{array}\right]$

For unknown mobile target positioning problem is converted into the above system of linear equations to solve the problem. Only when the matrix A reversible, the solution of equations $\mathrm{Ax}=\mathrm{b}$ is $\mathrm{x}=\mathrm{A}^{-1} b$. In order to get the only sure coordinates $\mathrm{X}$, you will need to meet $\mathrm{R}(\mathrm{A}, \mathrm{b})=3$, that means the type (1) at least has four lines. That is to say, to solve the unknown target location in the 3d environment coordinate, we need to set up at least four reference nodes in the same plane to determine the coordinates.

But in practice, because the measurement environment is not ideal, there will be certain error range. This will lead to four for measuring point as the centre, distance to the reference node is specific for the radius of the ball not intersect for a bit. And in the process of actual measurement, the interference and obstacles often appear all sorts of equipment. This will lead to the pending a point of reference for the mobile node can't receive all information, which will lead to type (3) the underdetermined and can't locate the target. Therefore, under normal circumstances, when do firemen positioning, it will set several reference nodes, make the type (3) for the overdetermined equations ${ }^{[8-10]}$.

When the type(3) become overdetermined equations, the solution of equations $\mathrm{Ax}=\mathrm{b}$ is not present. That will make the sum of the squares $\mathrm{J}=e^{T} e=(A x-b)^{T}(A x-b)$ of the error vector mode $e=A \hat{x}-b$ to the minimum. When the $\mathrm{J}$ takes the minimum, the solution is called the least squares solution $\hat{\mathrm{x}}_{L S}$. From the significance of the least squares, we know that the least-squares solution is unique, its expression is:

$$
\hat{x}_{L S}=\left(A^{T} A\right)^{-1} A^{T} b
$$

\section{Estimate the instantaneous coordinates of the under test moving target}

In the process of actual fire, the reference nodes detected by the mobile nodes will be less than 4 . At this point type(3) is under determined, the system couldn't get the under test moving target coordinates. But in actual fire rescue, as a result of environmental impact, the speed of firemen in the fire isn't particularly fast, and firefighters in general are moving as fast as possible. At the same time, the moving target positioning are often repeated. Accordingly, when it can't be located, we can set up the target trajectory model according to the results of previous target positioning the 
location of the trajectory. Then reuse the model to fit out the current time target location coordinates ${ }^{[10-12]}$.

The movement of firemen in the fire can be regarded as uniform or variable motion in several positioning times. When the system target failure, we can use previous motion speed, positioning time interval and a moment before the target location information to estimate the target location coordinates at the current moment.

Suppose the system positioning for $\mathrm{T}$, and the coordinates of the goals in each of the positioning time $\mathrm{t}_{0}, \mathrm{t}_{1}, \cdots, \mathrm{t}_{\mathrm{n}-1}, \mathrm{t}_{\mathrm{n}}, \mathrm{t}_{\mathrm{n}+1}, \cdots$ is $\left(\mathrm{x}_{0}, \mathrm{y}_{0}, \mathrm{z}_{0}\right), \quad\left(\mathrm{x}_{1}, \mathrm{y}_{1}, \mathrm{z}_{1}\right), \cdots,\left(\mathrm{x}_{\mathrm{n}-1}, \mathrm{y}_{\mathrm{n}-1}, \mathrm{z}_{\mathrm{n}-1}\right), \quad\left(\mathrm{x}_{\mathrm{n}}, \mathrm{y}_{\mathrm{n}}, \mathrm{z}_{\mathrm{n}}\right)$, $\left(x_{n+1}, y_{n+1}, z_{n+1}\right), \cdots$ respectively. The evaluation rate of the goal in the time $\left(t_{i-1}, t_{i}\right)(i=1,2,3, \cdots)$ within $\mathrm{x}, \mathrm{y}, \mathrm{z}$ axis direction is:

$$
\left\{\begin{array}{l}
v_{i x}=\frac{x_{i}-x_{i-1}}{T} \\
v_{i y}=\frac{y_{i}-y_{i-1}}{T} \\
v_{i z}=\frac{z_{i}-z_{i-1}}{T}
\end{array}\right.
$$

If the system locate failure at the time $t_{i}$, and positioning success at the time $\left(t_{i-5}, t_{i-1}\right)$, it is able to use the average speed of time $\left(\mathrm{t}_{\mathrm{i}-5}, \mathrm{t}_{\mathrm{i}-1}\right)$ as the movement speed of time $\left(\mathrm{t}_{\mathrm{i}-1}, \mathrm{t}_{\mathrm{i}}\right)$. And get the target velocity component of time $\left(\mathrm{t}_{\mathrm{i}-1}, \mathrm{t}_{\mathrm{i}}\right)$ along the $\mathrm{x}$ axis direction:

$$
\tilde{v}_{i}=\bar{v}_{i-1}=\frac{v_{i-1}+v_{i-2}+v_{i-3}+v_{i-4}}{4}
$$

Similarly we can work out the direction velocity component $\widetilde{v}_{i y}, \widetilde{v}_{i z}$ of time $\left(\mathrm{t}_{\mathrm{i}-1}, \mathrm{t}_{\mathrm{i}}\right)$ along the $\mathrm{y}$ axis and $\mathrm{z}$ axis. Postulate that the moving target coordinates is $\left(\mathrm{x}_{\mathrm{i}-1}, \mathrm{y}_{\mathrm{i}-1}, \mathrm{z}_{\mathrm{i}-1}\right)$ at the time $\mathrm{t}_{\mathrm{i}-1}$, then we can calculate the moving targets in time ti coordinates as follows:

$$
\left(\mathrm{x}_{\mathrm{i}}, \mathrm{y}_{\mathrm{i}}, \mathrm{z}_{\mathrm{i}}\right)=\left(\mathrm{x}_{\mathrm{i}-1}+\tilde{\mathrm{v}}_{\mathrm{ix}} \mathrm{T}, \mathrm{y}_{\mathrm{i}-1}+\tilde{\mathrm{v}}_{\mathrm{iy}} \mathrm{T}, \mathrm{z}_{\mathrm{i}-1}+\tilde{\mathrm{v}}_{\mathrm{iz}} \mathrm{T}\right)
$$

\section{The experimental results and analysis}

The simulation experiment about the movement of the firefighters is at a regular speed in the 35 * $65 \mathrm{~m}$ indoor. Because of the experimental conditions, the mobile node to be measured and the reference node environment are at the same horizontal plane. As a result, the $3 \mathrm{~d}$ coordinates can be degraded into two coordinates at this point, and the required number of reference nodes to the location should be reduce to 3 .

In the same horizontal plane, to $30 \mathrm{~m}$ direction for the $\mathrm{x}$ axis, $60 \mathrm{~m}$ for the $\mathrm{y}$ direction, establish xoy right Angle coordinate system. Select the coordinate system in the first quadrant of 10 random coordinates to be the reference node. The reference nodes are $1(720,2920)$, A2 $(1180,2680)$, A3(1200,110) , A4(1470,4340), A5(2250,440), A6(2510,870), A7(2540,5710), A8(2640,2260), $\mathrm{A} 9(2880,3510)$, A10(3000,1680) respectively. Under test target roughly along the $y=2 x$ direction, the positioning interval is $500 \mathrm{~ms}$, and record every moment target's actual location.

Because there may be some errors when positioning and measurement coordinates, when positioning in practice, moving target's actual coordinates is defined as the actual measured values with random error between $0 \sim 50 \mathrm{~cm}$. We can detect at least three reference nodes at every moment in the indoor basketball courts, so we will shut down the reference node detector at the positioning moment of 8,13,16,19,24,29 artificially. And estimate the coordinates by using the positioning data that success of target location at former four times. Compared the moving targets under actual coordinates with positioning or estimate coordinates of the system, such as 1 . 
Table 1 the comparison results of actual coordinates and moving target positioning system or estimate the coordinates

\begin{tabular}{|c|c|c|c|c|c|c|c|}
\hline time & $\begin{array}{c}\text { actual } \\
\text { coordinates }\end{array}$ & $\begin{array}{c}\text { Estimate the } \\
\text { coordinates }\end{array}$ & Deviation & time & $\begin{array}{c}\text { actual } \\
\text { coordinates }\end{array}$ & $\begin{array}{c}\text { Estimate the } \\
\text { coordinates }\end{array}$ & Deviation \\
\hline 1 & $(112,250)$ & $(94,300)$ & 53.14 & 16 & $(1602,3266)$ & $(1596,3208)$ & 58.31 \\
\hline 2 & $(180,472)$ & $(120,453)$ & 62.94 & 17 & $(1714,3444)$ & $(1731,3455)$ & 20.25 \\
\hline 3 & $(302,686)$ & $(294,631)$ & 55.58 & 18 & $(1806,3690)$ & $(1791,3704)$ & 20.52 \\
\hline 4 & $(388,870)$ & $(344,827)$ & 61.52 & 19 & $(1918,3858)$ & $(1955,3892)$ & 50.25 \\
\hline 5 & $(482,1058)$ & $(438,1036)$ & 49.19 & 20 & $(1994,4078)$ & $(2043,4033)$ & 66.53 \\
\hline 6 & $(618,1252)$ & $(569,1210)$ & 64.54 & 21 & $(2104,4254)$ & $(2122,4230)$ & 30 \\
\hline 7 & $(712,1432)$ & $(709,1484)$ & 52.09 & 22 & $(2214,4474)$ & $(2183,4431)$ & 53.01 \\
\hline 8 & $(792,1640)$ & $(769,1694)$ & 58.69 & 23 & $(2300,4684)$ & $(2296,4680)$ & 5.66 \\
\hline 9 & $(908,1856)$ & $(945,1846)$ & 38.33 & 24 & $(2386,4842)$ & $(2331,4878)$ & 65.73 \\
\hline 10 & $(1004,2048)$ & $(1035,2023)$ & 39.82 & 25 & $(2512,5070)$ & $(2533,5126)$ & 59.81 \\
\hline 11 & $(1114,2284)$ & $(1128,2307)$ & 26.93 & 26 & $(2594,5284)$ & $(2636,5246)$ & 56.64 \\
\hline 12 & $(1204,2482)$ & $(1216,2535)$ & 54.34 & 27 & $(2700,5472)$ & $(2668,5437)$ & 47.42 \\
\hline 13 & $(1292,2630)$ & $(1241,2661)$ & 59.68 & 28 & $(2818,5634)$ & $(2779,5627)$ & 39.62 \\
\hline 14 & $(1382,2862)$ & $(1407,2838)$ & 34.66 & 29 & $(2908,5850)$ & $(2932,5895)$ & 51 \\
\hline 15 & $(1484,3090)$ & $(1514,3139)$ & 57.45 & 30 & $(2998,6042)$ & $(2999,6086)$ & 44.01 \\
\hline
\end{tabular}

From table 1 we can see that moving target's actual coordinates and system defined coordinate both have high accuracy whether in system estimate or the coordinate measurement. All the observation time, the system minimum error as small as $5 \mathrm{~cm}$, the maximum error is less than $65 \mathrm{~cm}$, average error is only $47 \mathrm{~cm}$, error within the acceptable range.

\section{Conclusion}

This paper proposes a fireman positioning method based on RFID technology, which combined RSSI and TOF ranging in automatic positioning firefighters. When the system can locate, it determines target real-time coordinates with the least square method by setting the redundant reference node. When the system cannot locate, it determines the target coordinates by using the previous target coordinates and trajectory fitting method. By analyzing the experimental results, it can be found that the method can positioning in real time and accurately. The method has better performance in precision, computing speed and positioning cost. Therefore, the system has certain engineering use value, and it can be applied in fire firefighters positioning effectively.

\section{References}

[1] Wang Shouhui, Ceng Xiao Lin, chien-hung kuo, Meng Fankun. A new method of firefighters positioning [J]. Journal of information science and technology of China, 2012, (20) : 147-148

[2] Li Weifeng. The research of indoor localization based on RFID technology [D]. Master's degree thesis. Shanghai jiaotong university. 2010. 
[3] Wang Yile. The research and application of indoor locating method based on RFID [D]. Master's degree thesis. Xian university of science and technology, 2010.

[4] Zhao Gang, Liu Hongwu, Yang Guowei, Song Gaojun. Space partitioning virtual grid of RFID indoor localization algorithm [J]. Computer system application, 2013, 22 (1) : 83-86.

[5] Li Kun, Liu Zheng, Chen Xuyang. Firefighters positioning based on RFID [J]. Journal of electronics, 2015 (2) : 45

[6]Yuan-HungLee,Meng-YingTsai.A3DRFIDStaticTestSystemUsingaSphericalNear-FieldAntenna MeasurementChamber[J].AntennasandPropagation,2013,61(1):362-370

[7] Huang xiaodong, Liu Xiaodi, Ding Zhaoming. RSSI and TOF ranging technology and simulation study [J]. Journal of naval aeronautical engineering institute, 2012, 27 (5) : 567-570.

[8] Bao jianjun, Huo Zhenlong, Xu Hui etc. A high precision wireless mine personnel positioning algorithm [J]. Journal of industrial automation, 2009, 35 (10) : 18 to 21.

[9] Li Jing. The research of FID indoor three-dimensional localization algorithm [D]. University of electronic science and technology. 2013.

[10] Xu Suhong, Zhang Dongyang, She Qiurong etc. The study of moving target accurate positioning adaptive filter algorithm [J]. Journal of missiles and guided journal, 2003, 23 (1) : 125-127.

[11] Li Zhengdong, Zhang Kefan, Bao Jianjun. Underground moving target positioning method based on distance measurement technology [J]. Journal of industrial automation, 2015 (41) : 9-12.

[12] Chen jiao,Chen Wei, Lu Xiaoye, Gu WenLi. The study of GPSOne navigation and position calculated based localization algorithm [J]. Computer and digital engineering, 2012 (1) : 16 to 18 